\title{
A FAST METHOD FOR MEASURING THE SIMILARITY BETWEEN 3D MODEL AND 3D POINT CLOUD
}

\author{
Zongliang Zhang ${ }^{\mathrm{a}}$, Jonathan $\mathrm{Li}^{\mathrm{a}, * \mathrm{~b}}$, Xin $\mathrm{Li}^{\mathrm{c}}$, Yangbin Lin ${ }^{\mathrm{a}}$, Shanxin Zhang ${ }^{\mathrm{a}, \mathrm{d}}$, Cheng Wang ${ }^{\mathrm{a}}$ \\ ${ }^{a}$ Fujian Key Laboratory of Sensing and Computing for Smart Cities, Xiamen University, Xiamen, FJ 361005, China - \\ zhangzongliang@stu.xmu.edu.cn; \{junli, cwang\}@xmu.edu.cn; yblin.xmu@qq.com \\ ${ }^{\mathrm{b}}$ Mobile Mapping Lab, Department of Geography and Environmental Management, University of Waterloo, \\ Waterloo, ON N2L 3G1, Canada - junli@uwaterloo.ca \\ ${ }^{c}$ School of Electrical Engineering and Computer Science, Louisiana State University, Baton Rouge, LA 70803, USA - \\ xinli@1su.edu \\ ${ }^{\mathrm{d}}$ Xizang Key Laboratory of Optical Information Processing and Visualization Technology, Information Engineering College, \\ Xizang Minzu University, Xianyang, SX 712082, China - jaysean_sc@126.com
}

\section{Commission I/V, WG I/Va}

KEY WORDS: Partial Similarity, 3D Point Cloud, 3D Mesh, Laser Scanning, 3D Object Retrieval, Weighted Hausdorff Distance

\begin{abstract}
:
This paper proposes a fast method for measuring the partial Similarity between 3D Model and 3D point Cloud (SimMC). It is crucial to measure SimMC for many point cloud-related applications such as 3D object retrieval and inverse procedural modelling. In our proposed method, the surface area of model and the Distance from Model to point Cloud (DistMC) are exploited as measurements to calculate SimMC. Here, DistMC is defined as the weighted distance of the distances between points sampled from model and point cloud. Similarly, Distance from point Cloud to Model (DistCM) is defined as the average distance of the distances between points in point cloud and model. In order to reduce huge computational burdens brought by calculation of DistCM in some traditional methods, we define SimMC as the ratio of weighted surface area of model to DistMC. Compared to those traditional SimMC measuring methods that are only able to measure global similarity, our method is capable of measuring partial similarity by employing distance-weighted strategy. Moreover, our method is able to be faster than other partial similarity assessment methods. We demonstrate the superiority of our method both on synthetic data and laser scanning data.
\end{abstract}

\section{INTRODUCTION}

Measuring the similarity between 3D geometric objects plays an important role in many 3D applications such as 3D object retrieval (Biasotti et al., 2015) and inverse procedural modelling (Talton et al., 2011). Many methods have been proposed either for measuring the global similarity between two complete 3D objects, or for measuring the partial similarity between complete 3D object and incomplete structured 3D object (Savelonas et al., 2015). However, there are a few methods proposed for measuring the Similarity between 3D Model and 3D point Cloud (SimMC)

Measuring SimMC is becoming more and more important, as a result of easier and easier acquisition of point clouds due to the blooming of laser scanning techniques. A point cloud usually contains a large number of points. Point cloud is intrinsically incomplete and unstructured. SimMC is a special kind of partial similarity. The problem of partial similarity assessment is challenging and not being well solved (Sipiran et al., 2014).

In the field of Partial 3D Object Retrieval (P3DOR), some approaches have been presented for assessing the partial similarity in recent years. However, most of those approaches require structured data (e.g. mesh) as input (Lavoué, 2012) (Bronstein et al., 2011). Recently, a similarity assessment method applicable on point clouds was proposed in (Savelonas et al., 2016). The method calculates similarity by combining differential fast point feature histograms with Fisher encodings.
Although the similarity assessment methods introduced in the field of P3DOR can achieve state-of-the-art performance in terms of precison and recall, they may perform poorly in terms of speed. The size of model set used in P3DOR usually is not big. For example, the datasets used in (Savelonas et al., 2016) contain around 400 models. As a result, the time cost in P3DOR is not so important. However, in Inverse Procedural Modeling (IPM), we have to perform similarity assessment over procedural space which contains infinite models. The time cost for similarity assessment hence becomes critical in IPM.

In this papar, we present a fast SimMC assessment method which borrows the mean error idea from MESH (Aspert et al., 2002). However, different from MESH aiming to measure global similarity, in order to measure partial similarity, our method employs distance-weighted strategy to express different importance of different parts in assessed objects. Our method is as fast as MESH which is one of the fastest global similarity assessment methods. Our method therefore is faster than exsiting partial similarity assessment methods to some extent.

The rest of this paper is organized as follows. Section 2 presents some related shape similarity assessment methods. Section 3 presents our distance-weighted partial similarity assessment method. Section 4 presents experimental evaluation. Section 5 presents concluding remarks.

${ }^{*}$ Corresponding author 


\section{RELATED WORK}

\subsection{Weighted Hausdorff Distance}

There are many modified Hausdorff distance measures have been proposed for different purposes in various applications. In face recognition, by assuming different regions have different importance, several weighted Hausdorff distance measures have been proposed. Spatially eigen-weighted Hausdorff distance, which was proposed in (Lin et al., 2003), defines weighting function based on an eigenface which can effectively reflect the face structure. Edge eigenface weighted Hausdorff distance, which was proposed in (Tan et al., 2011), defines weighted function based on the eigenface of edge images.

\subsection{Partial Similarity between 3D Objects}

Partial similarity assessment between 3D objects is mostly studied in P3DOR which is essentially distinct from global 3D object retrieval. Persist heat signatures (Dey et al., 2010) was proposed for matching incomplete models. Bilateral map (van Kaick et al., 2013) was proposed as a local shape descriptor for partial matching. A pariwise 3D shape context for partial object retrieval was proposed in (Yu et al., 2014). A partial similarity assessment method based on differential fast point feature histograms and Fisher encodings was recently proposed in (Savelonas et al., 2016).

\section{METHOD}

We define the partial similarity between a 3D model and a 3D point cloud as the ratio of weighted surface area of the model to the weighted one-sided Hausdorff distance from the model to the point cloud.

\subsection{Symmetrical Hausdorff Distance}

The Symmetrical Hausdorff Distance (SHD) $d_{s}$ between two point sets $A$ and $B$ is defined as:

$$
d_{s}(A, B)=\max [d(A, B), d(B, A)]
$$

where $d(A, B)$ is One-sided Hausdorff Distance (OHD) from $A$ to $B$ :

$$
d(A, B)=\max _{a \in A} d(a, B)
$$

and

$$
d(a, B)=\min _{b \in B}\|a-b\|
$$

where $\|\cdot\|$ is Euclidean norm.

We can use SHD $d_{s}(A, B)$ to exactly assess the global similarity between point sets $A$ and $B$. $A$ and $B$ are identical if $d_{s}(A, B)$ equals 0 .

\subsection{Mean Error}

According to Eqs. (1) and (2), to calculate SHD , we have to compute OHD two times, one time from $A$ to $B$, another time from $B$ to $A$. (Aspert et al., 2002) proposes approximating SHD by Mean Error (ME) which only needs to compute OHD one time and therefore dramasttically saves time. The ME $d_{m}$ between surface $A$ and point set $B$ is defined as:

$$
d_{m}(A, B)=\frac{1}{|A|} \iint_{a \in A} d(a, B) d A
$$

where $|A|$ denotes the area of $A$.
Note that, to compute ME between two point sets, one of the point sets must be in structured form (e.g. surface), so as we can get the area of it. In practice, surface is commonly represented as discrete meshes (e.g. triangular meshes). Suppose $A$ consisting of $N$ non-overlapping sub-surfaces (meshes):

$$
A=\cup_{i=1}^{N} A_{i}
$$

Then the Discrete Mean Error (DME) $d_{d m}$ between $A$ and $B$ can be defined as:

$$
d_{d m}(A, B)=\frac{\sum_{i=1}^{N} d\left(A_{i}, B\right)\left|A_{i}\right|}{\sum_{i=1}^{N}\left|A_{i}\right|}
$$

\subsection{Partial Similarity}

Partial similarity is different from global similarity. The global similarity can be assessed by computing SHD or ME. However, it is not straightforward to assess partial similarity. If an object is a part of another object, or these two objects have common part, then these two objects are partially similar. As shown in Fig. 1, (a) and (b) are partially similar since (b) is a part of (a).

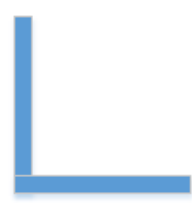

(a)

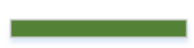

(b)

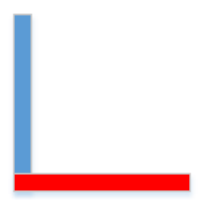

(c)
Figure 1: Illustration of partial similarity. (a) and (b) are two objects. The red part in (c) shows the overlap between (a) and (b). Actually, (b) is a part of (a).

\subsection{Reciprocal Weighted Mean Error}

We propose two kinds of Reciprocal Weighted ME (RWME) for assessing the partial similarity between two 3D objects. One is smoothly-RWME, another one is piecewise-RWME.

Given a surface $A$, which consists of $N$ sub-surfaces (see Eq. (5)), and a point set $B$, the RWME $r$ between $A$ and $B$ is defined as:

$$
r(A, B)=\frac{\sum_{i=1}^{N} w_{i}\left|A_{i}\right|}{c+\sum_{i=1}^{N} w_{i} d\left(A_{i}, B\right)}
$$

where $c$ is a positive number which is fixed to 0.1 in this paper, and $w_{i}$ is the weight.

For smoothly-RWME, $w_{i}$ is defined as:

$$
w_{i}=\exp \left(\frac{-d\left(A_{i}, B\right)}{h}\right)
$$

where $h$ is a positive number. In this paper, we set $h$ to 1 . And for piecewise-RWME, $w_{i}$ is defined as:

$$
w_{i}=\left\{\begin{array}{l}
1, d\left(A_{i}, B\right)<t \\
0, \text { otherwise }
\end{array}\right.
$$

where $t$ is a number as a threshold. 


\subsection{Computation of $\mathrm{OHD}$}

According to Eq. (7), to compute RWME between a surface and a point set, we have to compute OHD from the sub-surfaces of the surface to the point set, which consists of two parts. The first part is sampling points from the sub-surfaces, for which we adopt a uniform random sampling strategy. The second part is searching the point set for the nearest point of a query point, which is time consuming while the point set contains a large number of points (e.g. a laser scanning point cloud consisting of millions of points). We employ the FLANN (Muja and Lowe, 2014) algorithm to achieve the searching. The computational complexity for computing RWME depends on the number of points sampled from the surface and the size of the point set.

\section{EXPERIMENTAL RESULTS}

\subsection{Synthetic Data}

We test our method on a synthetic dataset consisting of 3 target models and 3 query point clouds. Fig. 2 shows the models $M_{1}$, $M_{2}$ and $M_{3}$. The height, width and length of the models are all $10 \mathrm{~m}, 20 \mathrm{~m}$ and $20 \mathrm{~m}$ respectively. $M_{1}$ and $M_{2}$ both have 4 faces. $M_{3}$ has 3 faces.

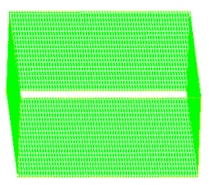

(a)

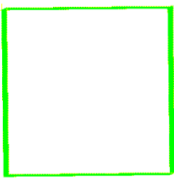

(d)

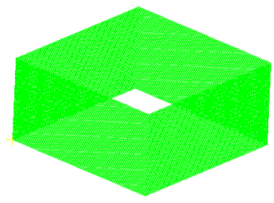

(b)

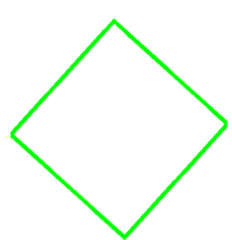

(e)

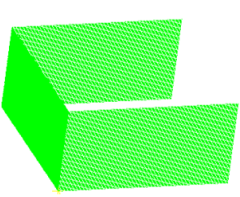

(c)

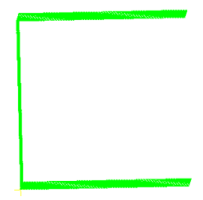

(f)
Figure 2: Models $M_{1}, M_{2}$ and $M_{3}$, from left to right. The top and bottom rows show the sideview and topview of the models respectively (the same below in this paper).

Figs. 3, 4 and 5 show the 3 query point clouds $C_{1}, C_{2}$ and $C_{3}$ (in blue). The figures also show the overlapping of the point clouds and the target models. Actually, $C_{1}, C_{2}$ and $C_{3}$ are point sets uniformly sampled from one face, two faces and three faces of $M_{1}$ respectively.

Table 1 shows the smoothly-RWMEs and DMEs (in italics) between models and point clouds. Bigger RWME indicates more partially similar, and smaller DME indicates more globally similar. In the computation of RWMEs and DMEs, we divide the models into their primitive faces. In other words, $N$ in Eqs. (7) and (4), the number of sub-surfaces of $M_{1}, M_{2}$ and $M_{3}$ are 4, 4 , and 3 resp.. From the table, investigating partial similarity RWME at first, we can see all the point clouds are dissimilar to $M_{2}$ and partially similar to $M_{1}$ and $M_{3}$, and can also see $C_{3}\left(C_{2}\right)$ is more partially similar to $M_{1}$ and $M_{3}$ than $C_{2}\left(C_{1}\right)$. Now investigating global similarity DME together, $C_{1}\left(C_{2}\right.$ or $\left.C_{3}\right)$ has almost the same partial similarity but different global similarity to $M_{1}$ or $M_{3}$. To sum up, the table shows the capability of our method for partial similarity assessement.

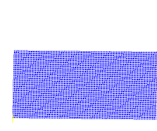

(a)

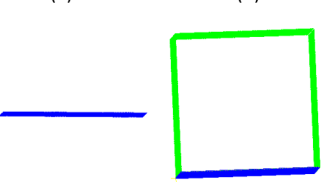

(e)

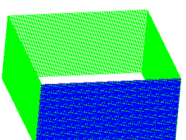

(b)

$$
\text { (f) }
$$

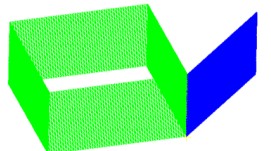

(c)

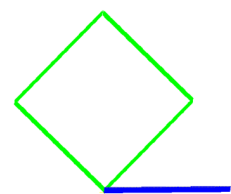

(g)

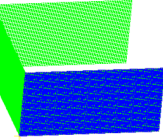

(d)

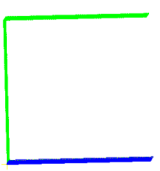

(h)
Figure 3: Point cloud $C_{1}$. The leftmost column shows $C_{1}$ alone. The remaining 3 columns show the overlapping of $C_{1}$ with $M_{1}$, $M_{2}$ and $M_{3}$, from left to right.

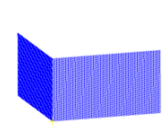

(a)

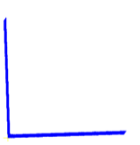

(e)

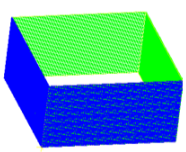

(b)

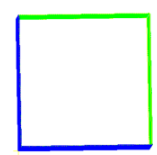

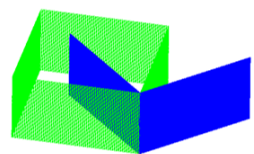

(c)

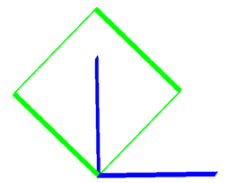

(g)

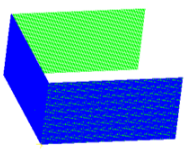

(d)

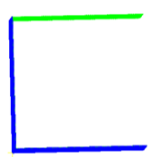

Figure 4: Point cloud $C_{2}$. The leftmost column shows $C_{2}$ alone. The remaining 3 columns show the overlapping of $C_{2}$ with $M_{1}$, $M_{2}$ and $M_{3}$, from left to right.

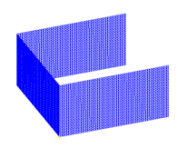

(a)

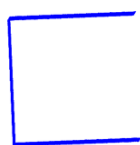

(e)

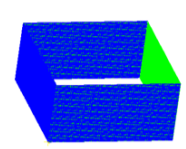

(b)

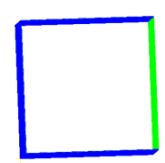

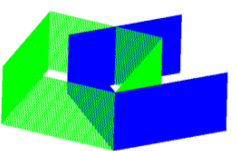

(c)

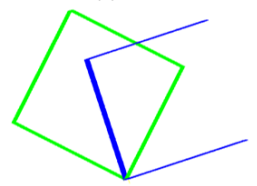

(g)

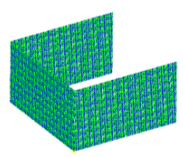

(d)

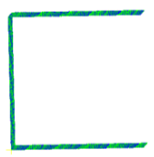

Figure 5: Point cloud $C_{3}$. The leftmost column shows $C_{3}$ alone. The remaining 3 columns show the overlapping of $C_{3}$ with $M_{1}$, $M_{2}$ and $M_{3}$, from left to right.

\subsection{Laser Scanning Point Cloud Data}

We also test our method on a mobile laser scanning point cloud $C_{4}$ which is scanned from a building and contains 190,677 points, as shown along with a 4-face cuboid model $M_{4}$ in Fig. 6. The time for computing RWMEs (in italics) between $M_{4}$ with varied sampling desities and $C_{4}$ with different filtering levels is showed in Table 2, in which \# $\left(M_{4}\right)$ denotes the number of points sampled from $M_{4}$ and $\#\left(C_{4}\right)$ denotes the number of points of $C_{4}$ after voxelized grid filtering. Apparently, we can get more accurate result of RWME while spending more time to take more points into account. The table shows the flexibility and stability of our method. 


\begin{tabular}{|l|c|c|c|}
\hline Model & $M_{1}$ & $M_{2}$ & $M_{3}$ \\
\hline Cloud & & & \\
$C_{1}$ & 803.407 & 0.0014 & 803.407 \\
& 15.0339 & 22.6779 & 13.3784 \\
$C_{2}$ & 1130.93 & 0.0058 & 1130.16 \\
& 10.0604 & 14.1425 & 6.7473 \\
$C_{3}$ & 1213.42 & 0.5834 & 1218.45 \\
& 2.5953 & 11.6424 & 0.1265 \\
\hline
\end{tabular}

Table 1: RWMEs and DMEs between models and point clouds.

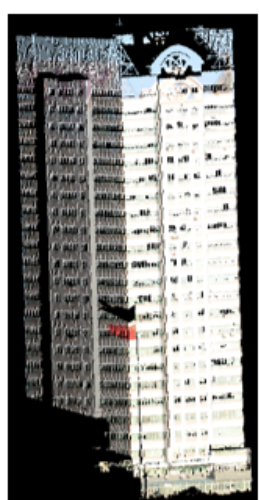

(a)

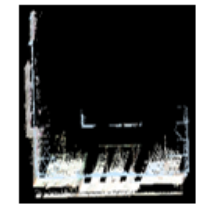

(d)

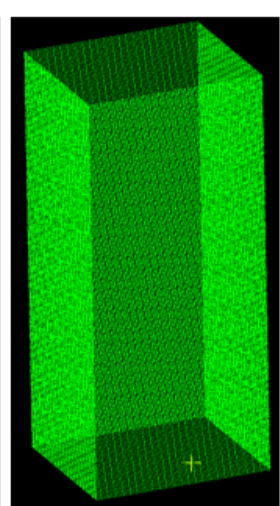

(b)

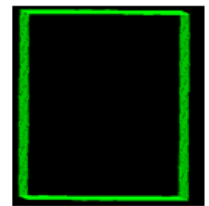

(e)

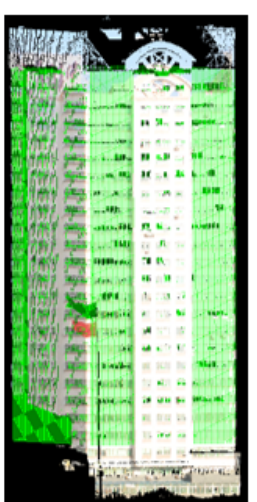

(c)

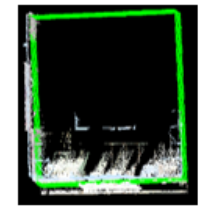

(f)
Figure 6: Point cloud $C_{4}$ (left), model $M_{4}$ (middle), and the overlapping of $C_{4}$ and $M_{4}$ (right).

\section{CONCLUDING REMARKS}

We presented an effective and flexible method for measuring the rigid partial similarity between a 3D model and a 3D point cloud. We defined the partial similarity as RWME which is the ratio of weighted surface area of the model to the weighted one-sided Hausdorff distance from the model to the point cloud. Different from other methods only available for assessing global similarity, our method is capable of assessing both global and partial similarity. Moreover, our method is able to be faster than other partial similarity assessment methods. The experiments for synthetic data and laser scanning data testified the superiority of our method.

\section{ACKNOWLEDGEMENTS}

This work is supported by the Xizang Minzu University scientific research project (No. 13myZP03) and Xizang Autonomous Region Natural science fund projects (No. 2015ZR-14-16).

\section{REFERENCES}

Aspert, N., Santa Cruz, D. and Ebrahimi, T., 2002. Mesh: measuring errors between surfaces using the Hausdorff distance. In: $\operatorname{ICME}(1)$, pp. 705-708.

Biasotti, S., Cerri, A., Bronstein, A. and Bronstein, M., 2015. Recent trends, applications, and perspectives in 3D shape similarity assessment. In: Computer Graphics Forum, Wiley Online Library.

\begin{tabular}{|l|c|c|c|c|}
\hline$\#\left(M_{4}\right)$ & 30 & 856 & 83,442 & $8,341,962$ \\
\hline$\#\left(C_{4}\right)$ & & & & \\
905 & 0.000137 & 0.001136 & 0.134565 & 10.4305 \\
& 838.711 & 622.308 & 535.706 & 524.699 \\
3,376 & $9.7 \mathrm{e}-05$ & 0.002858 & 0.199431 & 16.0824 \\
& 1791.86 & 1128.01 & 879.656 & 857.128 \\
11,758 & 0.000299 & 0.007747 & 0.336818 & 30.9319 \\
& 1785.82 & 1462.44 & 1048.82 & 885.532 \\
37,617 & 0.000535 & 0.014857 & 0.707125 & 67.9103 \\
& 2012.11 & 1600.59 & 1098.18 & 1027.73 \\
103,396 & 0.000595 & 0.028038 & 1.30579 & 126.228 \\
& 2020.34 & 1643.3 & 1190.02 & 1049.67 \\
190,667 & 0.000658 & 0.033435 & 1.8441 & 178.746 \\
& 1680.44 & 1217.65 & 1206.3 & 1041.67 \\
\hline
\end{tabular}

Table 2: The time (in seconds) for computing RWMEs between $M_{4}$ with varied sampling desities and $C_{4}$ with different filtering levels.

Bronstein, A. M., Bronstein, M. M., Guibas, L. J. and Ovsjanikov, M., 2011. Shape google: Geometric words and expressions for invariant shape retrieval. ACM Transactions on Graphics 30(1), pp. 1 .

Dey, T. K., Li, K., Luo, C., Ranjan, P., Safa, I. and Wang, Y., 2010. Persistent heat signature for pose-oblivious matching of incomplete models. In: Computer Graphics Forum, Vol. 29number 5, Wiley Online Library, pp. 1545-1554.

Lavoué, G., 2012. Combination of bag-of-words descriptors for robust partial shape retrieval. The Visual Computer 28(9), pp. 931-942.

Lin, K.-H., Lam, K.-M. and Siu, W.-C., 2003. Spatially eigenweighted Hausdorff distances for human face recognition. Pattern Recognition 36(8), pp. 1827-1834.

Muja, M. and Lowe, D. G., 2014. Scalable nearest neighbor algorithms for high dimensional data. Pattern Analysis and Machine Intelligence, IEEE Transactions on 36(11), pp. 2227-2240.

Savelonas, M. A., Pratikakis, I. and Sfikas, K., 2015. An overview of partial 3D object retrieval methodologies. Multimedia Tools and Applications 74(24), pp. 11783-11808.

Savelonas, M. A., Pratikakis, I. and Sfikas, K., 2016. Fisher encoding of differential fast point feature histograms for partial 3D object retrieval. Pattern Recognition.

Sipiran, I., Meruane, R., Bustos, B., Schreck, T., Li, B., Lu, Y. and Johan, H., 2014. A benchmark of simulated range images for partial shape retrieval. The Visual Computer 30(11), pp. 12931308.

Talton, J. O., Lou, Y., Lesser, S., Duke, J., Měch, R. and Koltun, V., 2011. Metropolis procedural modeling. ACM Transactions on Graphics 30(2), pp. 11.

Tan, H., Zhang, Y.-J., Wang, W., Feng, G., Xiong, H., Zhang, J. and Li, Y., 2011. Edge eigenface weighted Hausdorff distance for face recognition. International Journal of Computational Intelligence Systems 4(6), pp. 1422-1429.

van Kaick, O., Zhang, H. and Hamarneh, G., 2013. Bilateral maps for partial matching. In: Computer Graphics Forum, Vol. 32number 6, Wiley Online Library, pp. 189-200.

Yu, Y., Li, J., Yu, J., Guan, H. and Wang, C., 2014. Pairwise three-dimensional shape context for partial object matching and retrieval on mobile laser scanning data. Geoscience and Remote Sensing Letters, IEEE 11(5), pp. 1019-1023. 